\title{
Maximally Spatial-Disjoint Lightpaths in Optical Networks
}

\author{
M. Waqar Ashraf ${ }^{1}$, Sevia M. Idrus*2, Farabi Iqbal ${ }^{3}$ \\ 1,2,3 Department of Electrical Engineering, Universiti Teknologi Malaysia, Malaysia. \\ ${ }^{1}$ Department of Computer Engineering, Bahauddin Zakariya University Multan, Pakistan.
}

\begin{tabular}{l}
\hline \hline Article Info \\
\hline Article history: \\
Received Nov 8, 2017 \\
Revised Jan 12, 2018 \\
Accepted Feb 16, 2018 \\
\hline
\end{tabular}

Keywords:

Disaster-aware routing

Network reliability

Network survivability

Optical networks

\begin{abstract}
Lightpaths enable end-to-end all-optical transmission between network nodes. For survivable routing, traffic is often carried on a primary lightpath, and rerouted to another disjointed backup lightpath in case of the failure of the primary lightpath. Though both lightpaths can be physically disjointed, they can still fail simultaneously if a disaster affects them simultaneously on the physical plane. Hence, we propose a routing algorithm for provisioning a pair of link-disjoint lightpaths between two network nodes such that the minimum spatial distance between them (while disregarding safe regions) is maximized. Through means of simulation, we show that our algorithm can provide higher survivability against spatial-based simultaneous link failures (due to the maximized spatial distance).
\end{abstract}

Copyright $@ 2018$ Institute of Advanced Engineering and Science. All rights reserved.

\section{Corresponding Author:}

Sevia M. Idrus,

Department of Electrical Engineering,

Universiti Teknologi Malaysia, Malaysia.

P19a, Skudai, Johor Bahru, Johor-81300, Malaysia.

Email: sevia@fke.utm.my

\section{INTRODUCTION}

Optical networks provide highly scalable network connectivity, enabling huge aggregated data transfer over very long distances. The occurrences of natural and anthropogenic disasters can have a devastating impact on the topological connectivity of these networks. In 2006, the earthquakes in Taiwan has cut multiple fibers connecting Asia and North America at sixteen different places, reducing the internet capacity of Hong Kong and China by $100 \%$ and $74 \%$, respectively [1]. In 2008, the Mediterranean Sea fiber cuts caused the 70\% loss of Egypt's connections to the outside world and 50\% to 60\% loss of India's outbound connectivity on the westbound route [2]. In 2015, an earthquake affected the rural information and communication technology infrastructure and services in Nepal $[3,4]$, causing a lot of damage in the form of collapsing of houses, schools, access centers, transmission towers, and failures of fiber backhaul and microwave links.

Disjoint lightpaths (pair of primary and backup lightpaths between two network nodes) [5] may consist of spatially-close fibers, which can cause concurrent failure of both lightpaths in the event of a disaster. There are many reasons for which fibers can be spatially-closed. Occasionally, to avoid higher cost of digging ducts, different fibers may have been placed together using a single duct. Figure 1 shows an example of duct sharing as spatially-close overlapping fiber. Fiber endpoints may also be spatially-close due to existing infrastructures or geographical characteristics. Non-overlapping fibers may also be spatially-close due to the close vicinity of their ducts. Although disjointed lightpaths can be physically disjointed, the existence of spatially-close fibers within their fiber sets risks the lightpaths to be affected simultaneously by a disaster occurrence. Hence, increasing the minimum spatial distance of disjoint lightpaths is important for network operators in order for them to ensure the robustness of network services against disaster-based failures. 


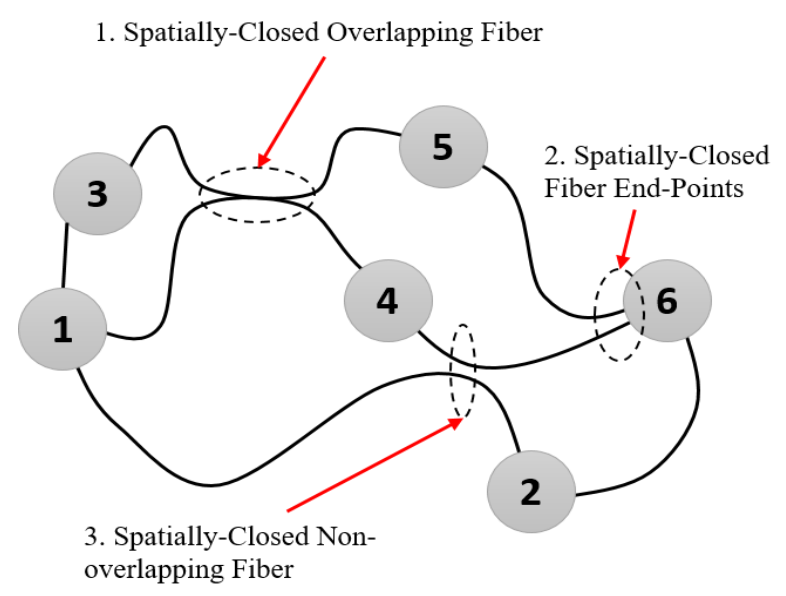

Figure 1. Types of Spatially-close Fibers

Figure 2 shows two link-disjoint paths $\{1 \rightarrow 2 \rightarrow 6\}$ and $\{1 \rightarrow 4 \rightarrow 6\}$, where fibers $1 \rightarrow 2$ and $4 \rightarrow$ 6 are spatially-close. Commonly, fibers are installed in non-straight manner between point-of-presences due to terrains, existing infrastructures and governing rules. We approximate fiber as a concatenation of multiple fiber segments of varying lengths, and can be mapped on geodetic co-ordinates (latitudes and longitudes) or translated into corresponding two-dimensional Cartesian co-ordinates. The remainder of the paper is as follows. Section II presents the related work. Section III provides the problem formulation and our proposed routing algorithm. Simulation results are presented in Section IV. Section V concludes the paper.

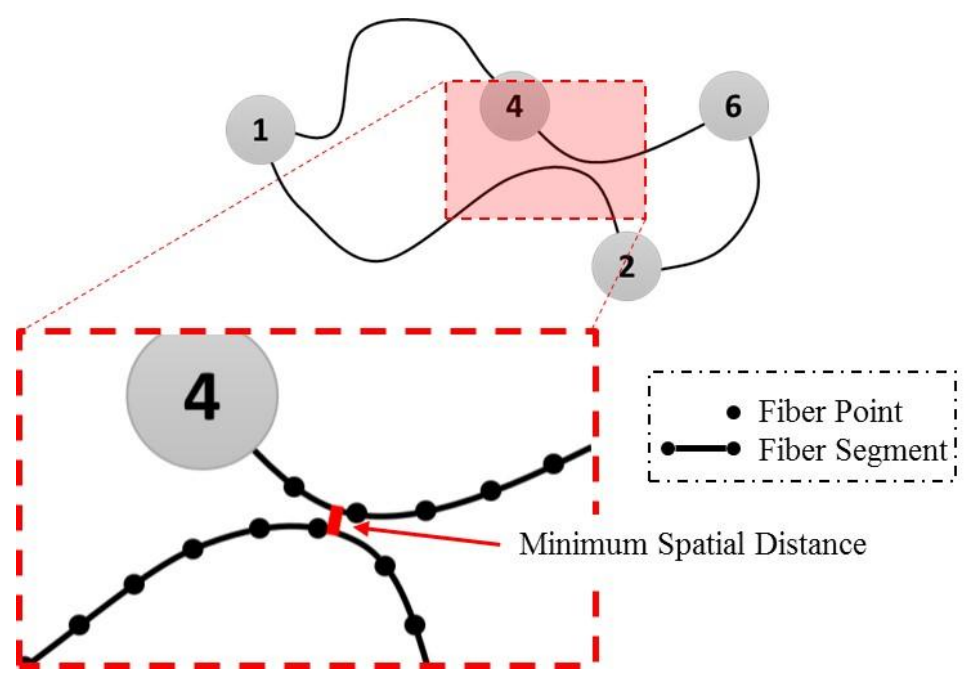

Figure 2. Example of a Pair of Link-disjoint Lightpaths with Minimum Spatial Distance

\section{RELATED WORK}

Disasters are inevitable, and can disrupt the network services significantly [6]. During the recent years, a lot of work has been conducted to address the issue of disaster vulnerabilities with different perspectives like natural disasters, electromagnetic pulse, weapons of mass destruction attacks, specific geographical locations and region-aware network augmentation to discourse the influence of a regional failures [7, 8-19]. The impact of failure events on network's services and capacity can be determined from the network geography. Dikbiyik et al. [9], proposed solutions to prevent connection failures from disasters through proactive approaches where damages and cascading failures are estimated as specific events like floods, hurricanes, earthquakes, and occurring in specific geographical locations. In [11], Neumayer et al. presented the geographic-based failure as generalized min-cut and max-flow problems, where a rival tries to 
reduce the network connectivity by multiple attacks. They proposed polynomial-time algorithm for the geographic min-cut problem and several approaches for the geographic max-flow problems.

Iqbal et al. [14] considered the geographical information of network nodes and links and include the notion of time-based disaster impact, for finding the risk profile of different network areas at different times. The rationale is that some disasters, like hurricanes, may traverse different network area at different times. They then provided polynomial-time algorithms to assess the most vulnerable network connections. Iqbal et al. [15] also proposed fast running algorithms for detecting spatially-closed fibers and grouping them using the minimum number of distinct risk groups. Agrawal et al. [16], proposed a scheme referred to as the Seismic Zone Aware Node Relocation (SZANR), based on the information on seismic zones by meteorological departments and other similar agencies. Results showed that significant enhancement in the network survivability can be attained in events of earthquakes through promptly minute changes in the network topology.

Sousa et al. [19] addressed a similar problem to this paper, the path geo-diversification problem, where a demand between two network nodes is supported by a pair of geographically-separated paths of a minimum distance. They proposed an ILP to solve the problem. In the same context, Wang et al. [20] presented a geographic aware route selection algorithm for finding alternative paths with appropriate geographical separation, referred to as the proximity factor. Contrary to their work, our proposed algorithm is based on the Yen's algorithm [21], such that the running time and accuracy of our algorithm can be tuned based on the selected $K$ value. ILP are often too time-consuming and our proposed algorithm provide the means for reducing the running time for finding a viable solution to the problem, albeit with lower optimality. However, when $k$ is unbounded, our proposed algorithm will always find the most optimal solution.

\section{PROBLEM FORMULATION AND PROPOSED ALGORITHM}

Let an undirected graph $G(N, L)$ represents a physical network, where $N=\left\{n_{1}, n_{2}, n_{3}, \cdots, n_{N}\right\}$ is the set of $|N|$ nodes and $L=\left\{l_{1}, l_{2}, l_{3}, \cdots, l_{M}\right\}$ is the set of $|M|$ undirected links representing bidirectional optical fibers. $\mathrm{P}=\left\{\mathrm{P}_{1}, \mathrm{P}_{2}, \mathrm{P}_{3}, \cdots, \mathrm{P}_{\mathrm{k}}\right\}$ is the set of candidate shortest lightpaths from source node $s$ to destination node $\boldsymbol{t}$ and $\mathrm{w}=\left\{\mathrm{w}_{1}, \mathrm{w}_{2}, \mathrm{w}_{3}, \cdots, \mathrm{w}_{\mathrm{k}}\right\}$ is the set of corresponding path weights. $\left(\mathrm{P}_{\mathrm{u}}, \mathrm{P}_{\mathrm{v}}\right)$ then represents the linkdisjoint pair of lightpaths with corresponding path weight pair $\left(\mathrm{w}_{\mathrm{u}}, \mathrm{w}_{\mathrm{v}}\right)$.

1. Provisioning of Disjoint Pair with Maximized Minimum Spatial Distance (DPMMSD) Problem:

2. Find a pair of link-disjoint lightpaths $\left(\boldsymbol{P}_{\boldsymbol{u}}, \boldsymbol{P}_{\boldsymbol{v}}\right)$ between two specific network nodes such that the minimum spatial distance between the lightpaths is maximized.

We proposed the DPMMSD algorithm in the context of the mentioned problem. The algorithm is divided into two parts. The first part of the algorithm finds $K$ number of shortest paths between nodes $s$ and $t$ in the given network (using Yen's algorithm [21]). Other k-shortest path routing algorithms may also be used instead. The second part of the algorithm proceeds from line 3 to line 11. Line 5 confirms whether a pair $\left(P_{u}, P_{v}\right)$ of shortest lightpaths is link-disjointed. If pair $\left(P_{u}, P_{v}\right)$ is link-disjointed, then the corresponding minimum spatial distance (msd) and the average minimum spatial distance (msdAvg) of the pair is computed in line 7 using Algorithm 3 (a modified form of the algorithm proposed in [15] to detect spatially-closed fibers, based on the use of k-d tree and $\mathrm{k}$ nearest neighbor search [22-24]). In some cases, multiple linkdisjoint pairs may have same maximum value of minimum spatial distance. Hence, we introduce another supporting decision variable, the average minimum spatial distance (msdAvg) that represents the quantitative disjointness between the lightpaths, as a tiebreaker. Line 8-11 selected the link-disjoint pair of lightpaths based on msd and msdAvg values with maximized minimum spatial distance.

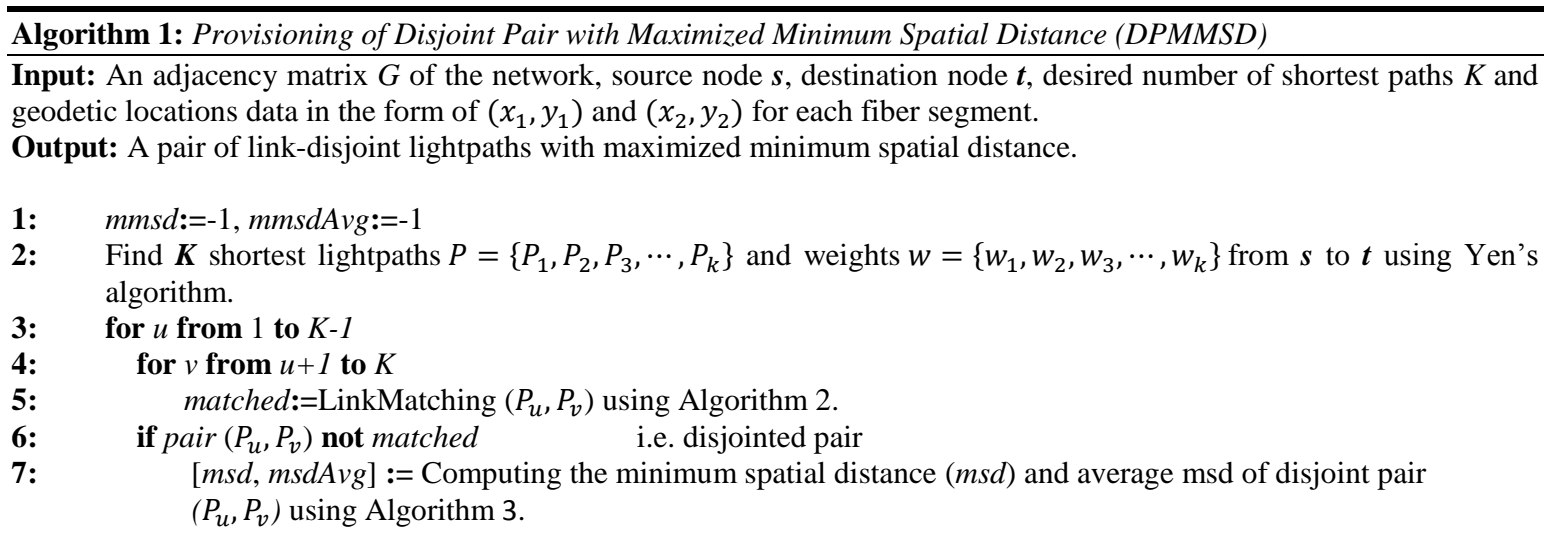


8: $\quad$ if $m m s d<m s d$ and $m m s d A v g<m s d A v g$

9: $\quad m m s d:=m s d$

10: $\quad m m s d A v g:=m s d A v g$

11: $\quad$ ConstrainedPair: $=\left(P_{u}, P_{v}\right)$
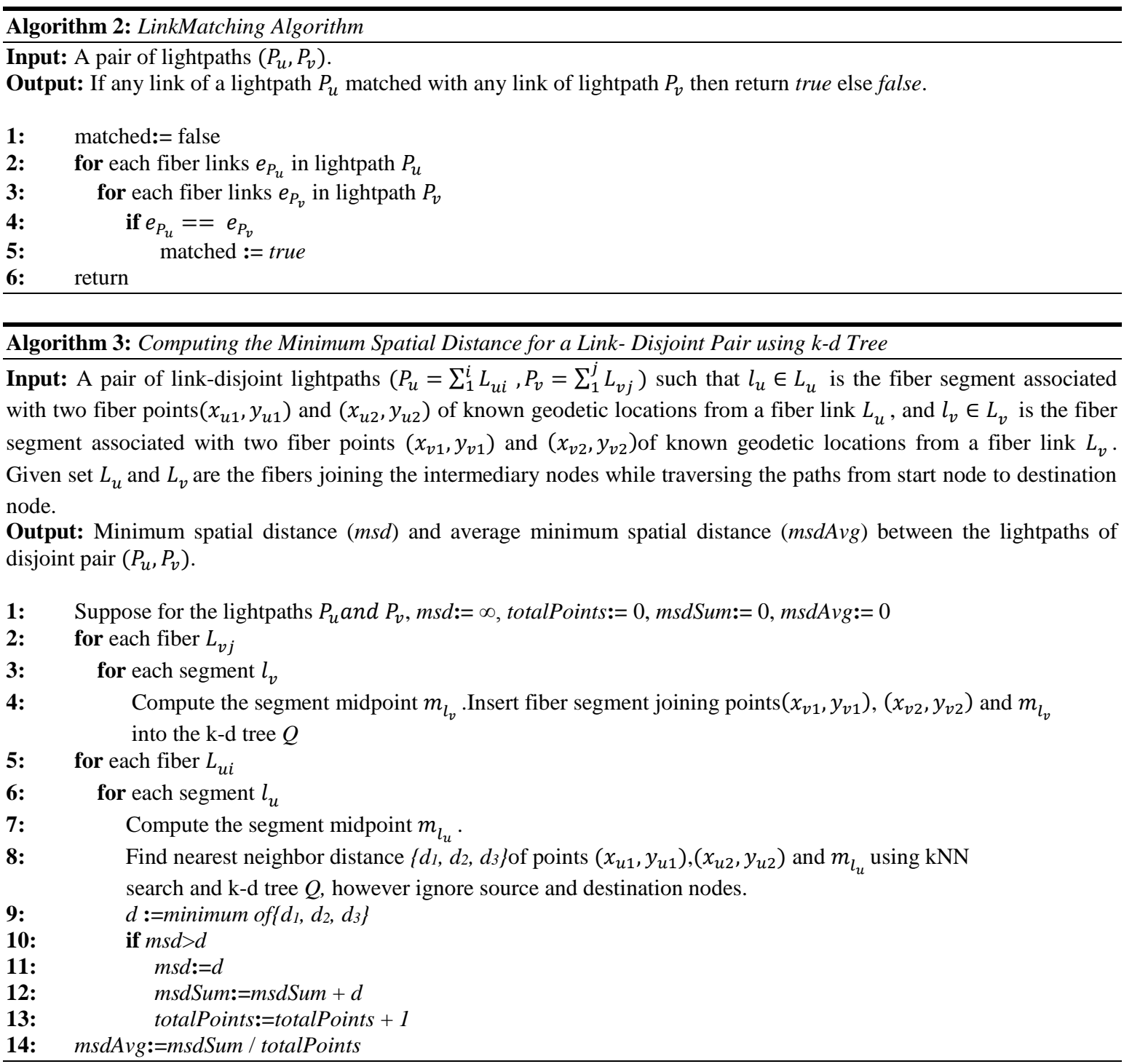

For geo-calculations over a great-circle (i.e. finding distance, mid-point, intermediate point etc.) between two latitude and longitude points, we used the formulae given in [25]. We also exclude certain fiber parts (within an exclusion distance from the source and the destination nodes) from the distance computation. Exclusion distance $(\delta)$ is the radius of the circular region for which the source node, destination node and enclosed fiber parts are assumed safe. The minimum spatial distance will be measured after this distance. The upper bound time complexity of Yen's Algorithm is $O\left(K N^{3}\right)$ [21] where $K$ is the number of shortest paths and $N$ is the number of nodes. Hence, the worst-case time complexity of our proposed algorithm is $O\left(K^{2} L^{2} S_{\max }^{2}\right)$, where $L$ is the number of total fiber links and $S_{\max }$ is the maximum number of fiber segments per fiber.

\section{SIMULATION RESULTS}

We simulate the performance of our algorithm using the European network. Performance and efficiency is analyzed while varying different parameters such as shortest paths $(K)$, exclusion distance from source and destination nodes $(\delta)$, computing time. The algorithm is coded in MATLAB R2016a and 
simulations are performed on $3^{\text {th }}$ Generation Intel® Core $15-3210 \mathrm{M} 2.5 \mathrm{GHz}$ machine of 6 GB RAM. All simulation results are averaged over 1000 runs.

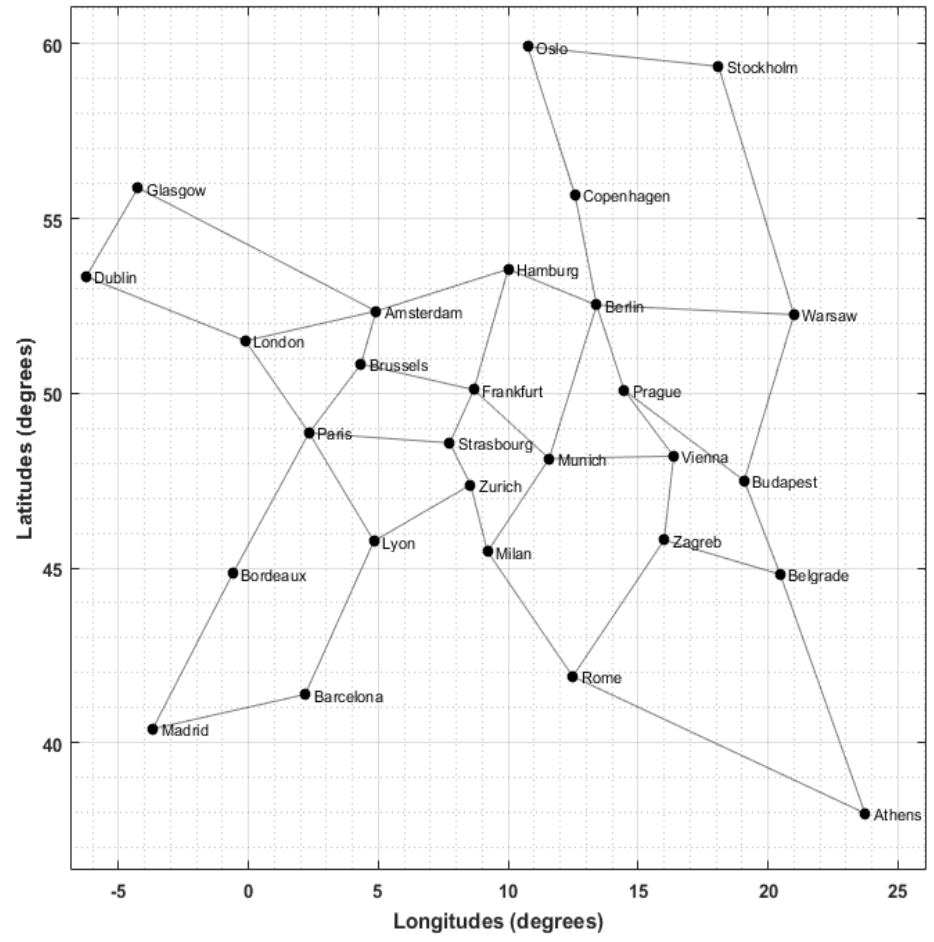

Figure 3: Geographically Mapped European Network

Figure 3 shows a representation of the European network [26], where nodes are mapped as per corresponding geodetic co-ordinates (latitudes and longitudes) in degrees.

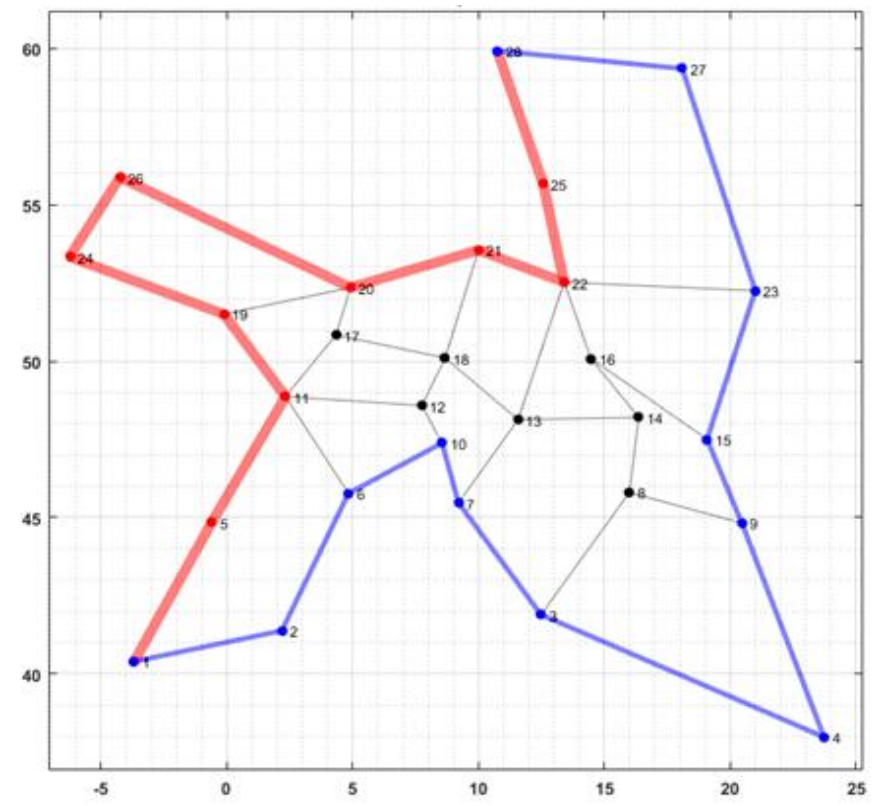

Figure 4: Graph of spatial-disjoint Pair with Minimum Spatial Distance of $223.42 \mathrm{~km}$ 
Figure 4 shows an example of a spatial-disjoint pair having minimum spatial distance $223.42 \mathrm{~km}$ between two lightpaths on the European network. Red and blue colored paths indicate primary path and backup path of the disjoint pair respectively.

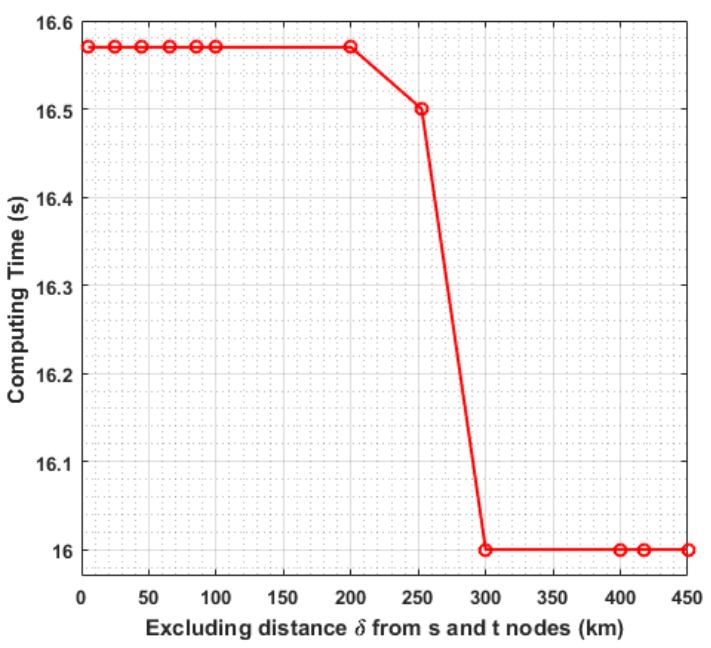

(a) Exclusion Distance vs Computing Time

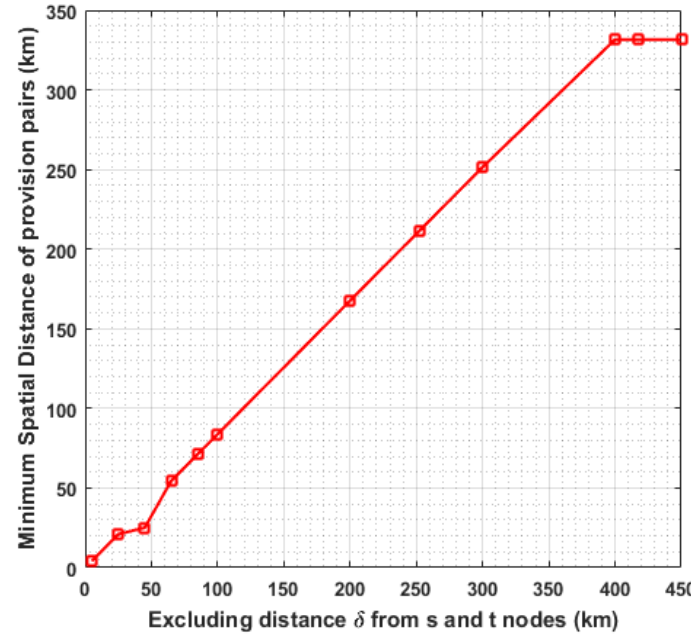

(b) Exclusion Distance VS Minimum Spatial Distance

Figure 5: Effect of Excluding Distance on Computing Time and Minimum Spatial Distance

Figure 5(a) shows the declining impact of $\delta$ on the computing times for our proposed algorithm in European network. As we increased $\delta$, more fiber segments are ignored from the measurement of minimum spatial distance, reducing the computation time. Similarly, $\delta$ also affects the minimum spatial distance as shown in Figure 5(b). When $\delta$ is increased to a certain value, then the minimum spatial distance becomes constant.

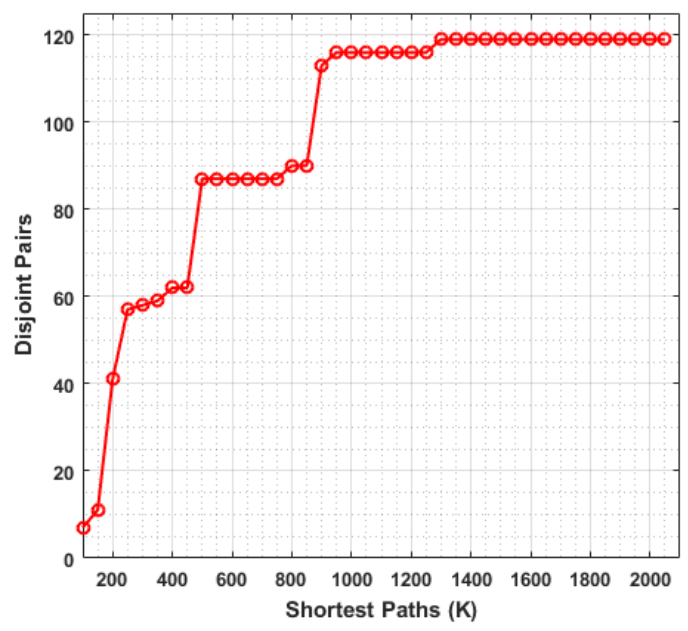

(a) Shortest Paths vs Disjoint Pairs

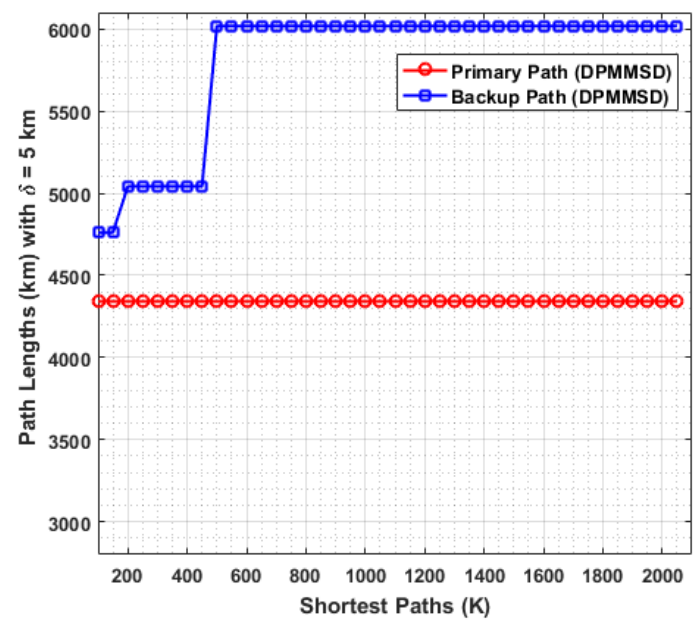

(b) Shortest Paths vs Path Lengths

Figure 6: Effect of K on Computation of Disjoint Pairs and Path lengths when $\delta=5 \mathrm{~km}$ 
The effect of the number shortest paths $(K)$ on the computation of disjoint pairs and path lengths for our algorithm is shown in Figure 6(a). The total number of disjoint path pairs depends on the total nodes and fiber links within the network. Figure 6(b) shows the variation of primary and backup path lengths by changing $K$ in the algorithm. The path becomes lengthy since DPMMSD provisioned a disjoint lightpath pair without considering path lengths (path lengths are not the main objective of the algorithm, which is maximizing the minimum spatial distance). During simulation, it was observed that most of the running time is consumed at finding and computing disjoint path pairs and their spatial distance. The processing time rises almost linearly when $K$ is increased. For the network, the maximum value of obtained $K$ is 2037 (119 linkdisjoint path pairs). The computation time will be constant when all possible $K$ and disjoint pairs have been obtained and no further pair can be found by increasing $K$.

\section{CONCLUSION}

In this paper, we have proposed a routing algorithm for provisioning a pair of link-disjoint lightpaths between two network nodes with maximum value of minimum spatial distance. Through simulation, we have shown that our algorithm can increase network survivability against spatial-based concurrent fiber failures, albeit with higher path weights. Maximally spatial-disjoint lightpaths have higher survivability against failing simultaneously in the event of spatial-based disaster occurrences on the physical plane. We have also tested the performance of our algorithm using a real-life optical network topology, where the running time and accuracy of our proposed algorithm can be tuned based on the number of considered shortest paths.

\section{ACKNOWLEDGEMENT}

This work was supported by Ministry of Higher Education Malaysia (MOHE) and the administration of Universiti Teknologi Malaysia through Institute Grant vote number 02K85.

\section{REFERENCES}

[1] J. P. Sterbenz, D. Hutchison, E. K. Çetinkaya, A. Jabbar, J. P. Rohrer, M. Schöller, et al., "Resilience and survivability in communication networks: Strategies, principles, and survey of disciplines," Computer Networks, vol. 54, pp. 1245-1265, 2010.

[2] J. Borland. Analyzing the internet collapse, MIT Technology Review, 2008. Available: http://www.nrcc.cornell.edu/page_ccd.html

[3] Seismonepal website. Available: http://seismonepal.gov.np/

[4] B. R. Dawadi and S. Shakya, "ICT Implementation and Infrastructure Deployment Approach for Rural Nepal," in Recent Advances in Information and Communication Technology 2016, ed: Springer, 2016.

[5] F Iqbal and F.A. Kuipers, "Disjoint paths in networks", Wiley Encyclopaedia of Electrical and Electronics Engineering, 2015.

[6] J. Rak, D. Hutchison, E. Calle, T. Gomes, M. Gunkel, P. Smith, et al., "RECODIS: Resilient Communication Services Protecting End-user Applications from Disaster-based Failures," in 2016 18th International Conference on Transparent Optical Networks (ICTON), 2016.

[7] S. Trajanovski, F. A. Kuipers, A. Ilić, J. Crowcroft, and P. Van Mieghem, "Finding critical regions and regiondisjoint paths in a network," IEEE/ACM Transactions on Networking (TON), vol. 23, pp. 908-921, 2015.

[8] F. Dikbiyik, A. S. Reaz, M. De Leenheer, and B. Mukherjee, "Minimizing the disaster risk in optical telecom networks," in Optical Fiber Communication Conference, 2012.

[9] F. Dikbiyik, M. Tornatore, and B. Mukherjee, "Minimizing the risk from disaster failures in optical backbone networks," Journal of Lightwave Technology, vol. 32, pp. 3175-3183, 2014.

[10] P. K. Agarwal, A. Efrat, S. K. Ganjugunte, D. Hay, S. Sankararaman, and G. Zussman, "The resilience of WDM networks to probabilistic geographical failures," IEEE/ACM Transactions on Networking (TON), vol. 21, pp. 1525-1538, 2013

[11] S. Neumayer, A. Efrat, and E. Modiano, "Geographic max-flow and min-cut under a circular disk failure model," Computer Networks, vol. 77, pp. 117-127, 2015.

[12] S. Neumayer and E. Modiano, "Network reliability with geographically correlated failures," in INFOCOM, 2010 Proceedings IEEE, 2010.

[13] B. Mukherjee, M. Habib, and F. Dikbiyik, "Network adaptability from disaster disruptions and cascading failures," IEEE Communications Magazine, vol. 52, pp. 230-238, 2014.

[14] F. Iqbal and F. Kuipers, "Spatiotemporal risk-averse routing," in 2016 IEEE Conference on Computer Communications Workshops (INFOCOM WKSHPS), 2016.

[15] F. Iqbal, S. Trajanovski, and F. Kuipers, "Detection of spatially-close fiber segments in optical networks," 2016 12th International Conference on the Design of Reliable Communication Networks (DRCN), 2016.

[16] A. Agrawal, P. Sharma, V. Bhatia, and S. Prakash, "Survivability Improvement Against Earthquakes in Backbone Optical Networks Using Actual Seismic Zone Information," arXiv preprint arXiv: 1703.02358, 2017. 
[17] Y. Awaji, H. Furukawa, S. Xu, M. Shiraiwa, N. Wada, and T. Tsuritani, "Resilient optical network technologies for Catastrophic Disasters," Journal of Optical Communications and Networking, vol. 9, pp. A280-A289, 2017.

[18] C. Galdamez and Z. Ye, "Resilient virtual network mapping against large-scale regional failures," in Wireless and Optical Communication Conference (WOCC), 2017.

[19] A. de Sousa, D. Santos, and P. Monteiro, "Determination of the Minimum Cost Pair of D-Geodiverse Paths," in 13th International Conference Design of Reliable Communication Networks, 2017.

[20] J. Wang, J. Bigham, and C. Phillips, "A Geographical Proximity Aware Multi-path Routing Mechanism for Resilient Networking," IEEE Communications Letters, 2017.

[21] J. Y. Yen, "Finding the k shortest loopless paths in a network," Management Science, vol. 17, pp. 712-716, 1971.

[22] J. L. Bentley, "Multidimensional binary search trees used for associative searching," Communications of the $A C M$, vol. 18, pp. 509-517, 1975.

[23] J. H. Friedman, J. L. Bentley, and R. A. Finkel, "An algorithm for finding best matches in logarithmic expected time," ACM Transactions on Mathematical Software (TOMS), vol. 3, pp. 209-226, 1977.

[24] R. Panigrahy, "An improved algorithm finding nearest neighbor using kd-trees," LATIN 2008: Theoretical Informatics, pp. 387-398, 2008.

[25] Calculate distance, bearing and more between Latitude/Longitude points. Available: http://www.movabletype.co.uk/scripts/latlong.html

[26] S. Verbrugge, D. Colle, P. Demeester, R. Huelsermann, and M. Jaeger, "General availability model for multilayer transport networks," in 5th International Workshop on Design of Reliable Communication Networks (DRCN 2005), 2005. 